\title{
Silver-Doped Layers of Implants Prepared by Pulsed Laser Deposition
}

\author{
Tomas Kocourek $^{1,2}$, Miroslav Jelinek ${ }^{1,2}$, Jan Miksovsky ${ }^{1,2}$, Karel Jurek ${ }^{1}$, Zdenek Cejka ${ }^{3}$, \\ Jaromir Kopecek $^{1}$
}

${ }^{1}$ Institute of Physics AS CR, Na Slovance 2, 18221 Praha 8, Czech Republic; ${ }^{2}$ Czech Technical University in Prague, Faculty of Biomedical Engineering, nam. Sitna 3105, 27201 Kladno, Czech Republic; ${ }^{3}$ ProSpon s.r.o.,J. Voskovne 3206, Kladno, Czech republic. Email: jelinek@fzu.cz

Received August 2013

\begin{abstract}
Physical and mechanical properties of silver-doped layers of titanium alloy Ti6Al4V and 316L steel prepared by pulsed laser deposition were studied. Metallic silver-doped coatings could be a new way for antibacterial protection in medicine. Thin films of silver and silver-doped materials were synthesized using $\mathrm{KrF}$ excimer laser deposition. The material was ablated from two targets, which composed either from titanium alloy with silver segments or from steel with silver segments. The concentration of silver ranged from 1.54 at.\% to 4.32 at.\% for steel and from 3.04 at.\% to 13.05 at.\% for titanium alloy. The layers properties such as silver content, structure, and adhesion were measured. Adhesion was studied using scratch test.
\end{abstract}

Keywords: Thin Layer; Silver; Titanium Alloy; Steel; Pulsed Laser Deposition; Adhesion; Implant

\section{Introduction}

One of the most serious complications of surgical treatment of fractures is infections. The infectious complications prolong healing and prevent fractures healing deplete the body's immune system. The solution usually requires repeated surgeries and multiplies healing costs and inconvenience for patients. The aim of this work is to find method to create antibacterial materials that will reduce the possibility of an infection or severity of infectious complications in patients after the surgical treatment of fractures. The use of fixation screws for temporary immobilization of broken bones entails considerable risk of infection due to the possibility of the bacteria spread along the outside surface of the fixing screws into the body (see Figure 1). Since the silver is known for its excellent antibacterial properties, the use of it as a suitable dopant seems like viable road to take. Coated of implants was given by pulse laser deposition. Silver concentration for various deposition conditions, films structure and adhesion were also studied [1,2].

\section{Experimental}

Deposition. Silver-doped layers of titanium alloy Ti6Al4V and 316L steel were prepared by PLD using a $\mathrm{KrF}$ excimer laser $(\mathrm{l}=248 \mathrm{~nm}, \mathrm{t}=20 \mathrm{~ns}$, rep. rate of 10
$\mathrm{Hz}$ ) (see Figure 2). The laser beam was focused on a silver target with energy density of $2 \mathrm{Jcm}^{-2}$, silver with Ti6Al4V or silver with 316L steel targets with energy density of $5 \mathrm{Jcm}^{-2}$. Material was ablated from one target

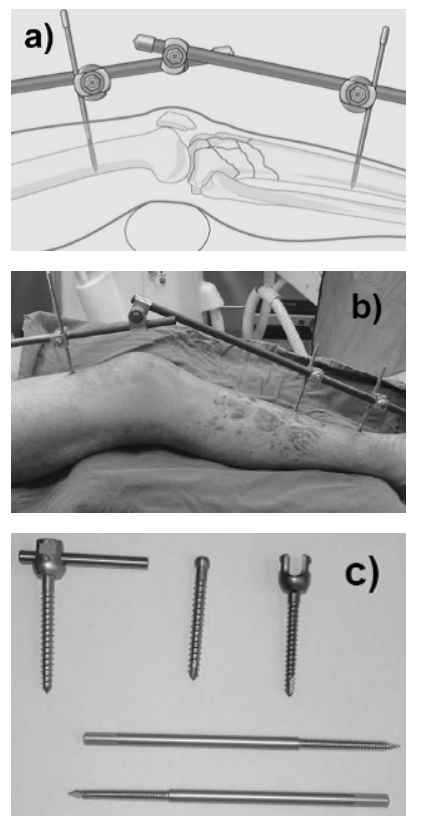

Figure 1. (a) Scheme of application of fixation screws; (b) Photo of application of fixation screws; (c) Fixation screws. 


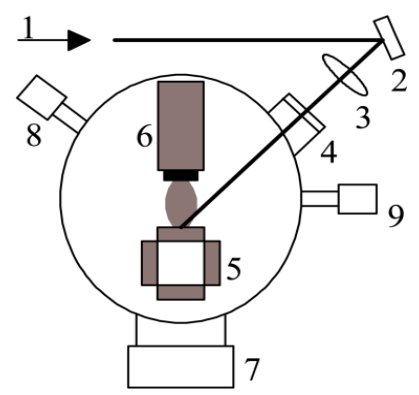

Figure 2. The basic scheme of the experimental apparatus for PLD: (1) laser beam, (2) mirrors, (3) focusing lens, (4) quartz window, (5) target holder, (6) substrate holder, (7) vacuum pump, $(8,9)$ Pirani and Penning vacuum gauges, respectively.

composed from silver and titanium alloy or steel segments. Substrate (Ti6Al4V, 316L steel or Si (100)) was $35 \mathrm{~mm}$ away from target. Substrate was held at room temperature. Films were grown in argon atmosphere of $0.25 \mathrm{~Pa}$. The substrates were cleaned by RF discharge before deposition process.

Thickness and roughness was measured by Alphastep IQ mechanical profilometer (KLA Co.).

Concentration of silver was determined using WDX measurement (WDX — wavelength dependence X-ray analysis) was analyzed with EDAX Jeol Supersprobe 733.

Structure of layers was determined by XRD in parallel beam geometry and detector scan with stationary sample and glazing angle of incidence (GAOI) were used.

Adhesion. For the adhesion measurements we used macro scratch tester REVETEST (CSM Instruments co.).

\section{Results and Discussion}

Thickness of PLD created silver layers was $100 \mathrm{~nm}$ and $350 \mathrm{~nm}$ and thickness of PLD created silver-doped layers was from $94 \mathrm{~nm}$ to $398 \mathrm{~nm}$, depending on target, see in Table 1.

Roughness of silver-doped 316L steel films was from $12 \mathrm{~nm}$ to $29 \mathrm{~nm}$ and the roughness of silver-doped titanium alloy Ti6Al4V films was from $5 \mathrm{~nm}$ to $28 \mathrm{~nm}$, see in Table 1.

Silver concentration - WDX measurement confirmed the increasing concentration of silver with increasing segment of the silver piece on the target - during deposition process. For the layers of 316L steel doped by silver amount of silver is more complicated, see in Table 1. of PLD created silver layers was $100 \mathrm{~nm}$ and $350 \mathrm{~nm}$ and thickness of PLD created silver-doped layers was from $94 \mathrm{~nm}$ to $398 \mathrm{~nm}$, depending on target, see in Table $\mathbf{1}$. The concentration of silver ranged from 1.54 at.\% to 4.32 at.\% for steel and from 3.04 at.\% to 13.05 at.\% for titanium alloy. It confirmed the increasing concentration of silver with increasing size of silver target during the deposition process. This increase is clear for titanium alloy
Table 1. The deposition condition for fabrication of silverdoped thin films by PLD process, the thickness, roughness and concentration of silver in the layers.

\begin{tabular}{|c|c|c|c|c|c|}
\hline Sample & Substrate & $\begin{array}{c}\text { Roughness } \\
\text { Ra [nm] }\end{array}$ & $\begin{array}{c}\text { Thickness } \\
\text { [nm] }\end{array}$ & Target (Size) & $\begin{array}{c}\text { At.[\%] } \\
\text { of Ag }\end{array}$ \\
\hline Ag-1 & Ti6Al4V & - & 100 & Ag & 100 \\
\hline Ag-2 & Ti6Al4V & - & 350 & $\mathrm{Ag}$ & 100 \\
\hline S-1 & 316L steel & 13 & 161 & 316L steel: Ag (40:1) & 1.54 \\
\hline S-2 & 316L steel & 29 & 260 & 316L steel: Ag (20:1) & 4.32 \\
\hline S-3 & 316L steel & 12 & 94 & 316L steel: Ag (10:1) & (3.53) \\
\hline T-1 & Ti6Al4V & 5 & 398 & Ti6Al4V: Ag (40:1) & 3.04 \\
\hline T-2 & Ti6Al4V & 28 & 294 & Ti6Al4V: Ag (20:1) & 5.05 \\
\hline T-3 & Ti6Al4V & 25 & 198 & Ti6Al4V: Ag (10:1) & 13.05 \\
\hline
\end{tabular}

layers. For the layers of 316L steel doped by silver the amount of silver concentration dependence is not monoteos and is more complicated, see in Table $\mathbf{1 .}$

Crystallinity. In the XRD spectrum of silver-doped 316L steel films were identified peak of intermetallic compounds (compound silver with $\mathrm{Fe}, \mathrm{Cr}, \mathrm{Ni}$, and $\mathrm{Mo}$ ). In the XRD spectrum of the titanium alloy doped by silver there are no new peaks, see in Figures 3(a) and (b).

Adhesion. For samples Ag-1 and Ag-2 of pure silver layers we used linear progressive scratch with initial load $1 \mathrm{~N}$ and the end load $5 \mathrm{~N}$. Loading rate was $4 \mathrm{~N} / \mathrm{min}$. Length of the scratches were $8 \mathrm{~mm}$. Two scratches were performed on each sample. The layers were very soft and were penetrated at the start by initial load. For sample Ag-1 there was no delamination observed. For sample Ag-2 we observed delamination for critical force approximately 1.75 N, see in Figure 4. Pure silver has low adhesion.

For samples S-1, S-2, S-3, T-1, T-2, and T-3 we used linear progressive scratch with initial load $1 \mathrm{~N}$ and the end load $30 \mathrm{~N}$. Loading rate was $15 \mathrm{~N} / \mathrm{min}$. Length of the scratches were $5 \mathrm{~mm}$. Two scratches were performed on each sample. Samples were tested for two various roughnesses of substrates, polished and lathed. Both substrates had similar behavior. We did not observe any penetration through layer or delamination. The behavior of the samples was similar to bulk material, see in Figures 5(a) and (b).

\section{Conclusion}

The metallic (titanium alloy Ti6Al4V and 316L steel) layers with various concentration of silver were prepared by PLD. Composition was determined by WDX. The amount of silver in Ti6Al4V layers was from 3.04 to 13.05 at.\%. The amount of silver in 316L steel layers was from 1.54 to 4.32 at.\%. Minor changes were observed in 


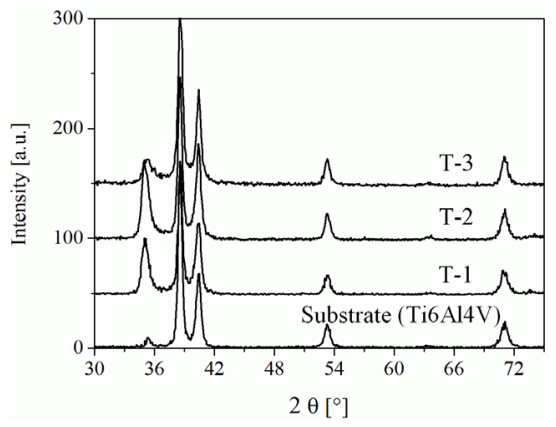

(a)

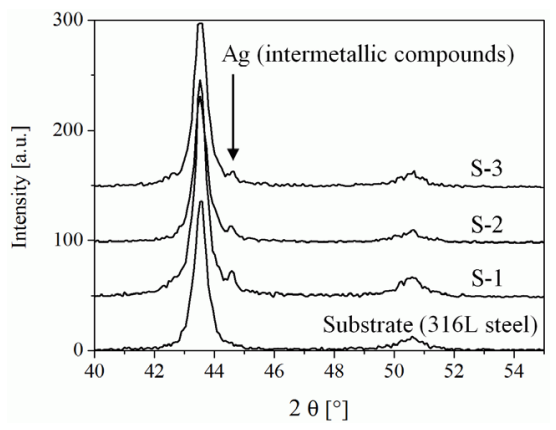

(b)

Figure 3. (a) XRD spectra of silver-doped 316L steel layers on 316L steel substrate; (b) XRD spectra of silver-doped titanium alloy Ti6Al4V layers on titanium alloy Ti6Al4V substrate.
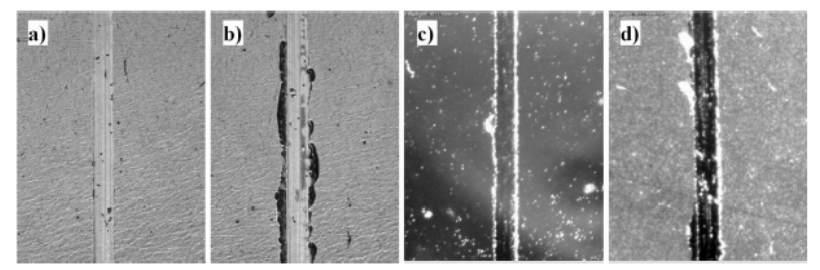

Figure 4. Example of delamination of $350 \mathrm{~nm}$ thick silver layer (Ag-2) load 1.4 N (a) and load $1.75 \mathrm{~N}$ (b). Comparison of adhesion for $100 \mathrm{~nm}$ (Ag-1) (c) and $350 \mathrm{~nm}$ (Ag-2) (d) thick silver layers for the force of $4 \mathrm{~N}$.

crystalline structure of the doped steel, which can be assigned to intermetallic compounds with silver. We did not observe new peaks in XRD spectrum of the titanium alloy doped by silver. No silver crystalline phase was found. All layers surfaces were covered with droplets. The adhesion of the silver-doped 316L steel and Ti6Al4V
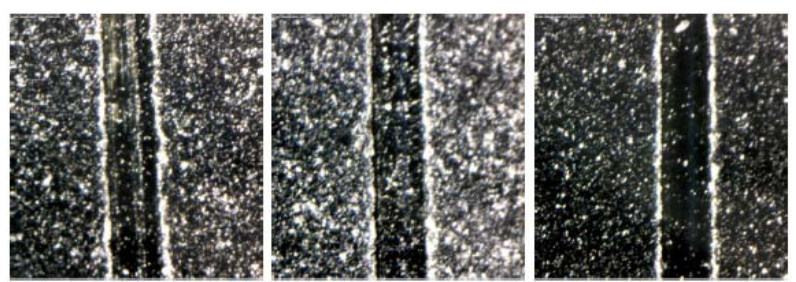

(a)
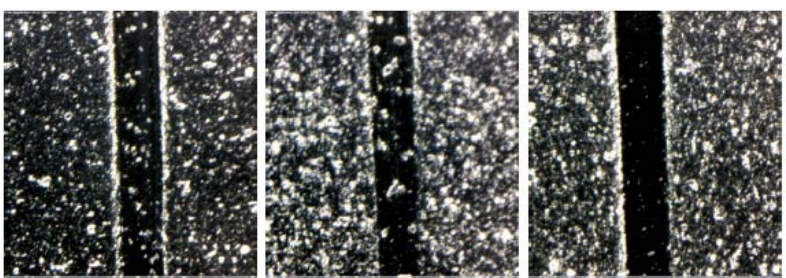

(b)

Figure 5. (a) Example of macro scratch on the layers of Ag doped steel 316L. From left sample S-1, S-2 and S-3 (load for all samples $24 \mathrm{~N}$ ); (b) Example of macro scratch on the layers of Ag doped Ti6Al4V alloy. From left sample T-1, T-2 and T-3 (load for all samples $24 \mathrm{~N}$ ).

was outstanding. We did not observe any delamination of layers. The transition between the layer and substrate was not observed.

\section{Acknowledgements}

The project has been supported by Czech Technical University CTU Prague No. SGS 12/167/OHK4/2T/17, grant FR- TI 3/088, grant COST LD 12068 and grant KAN300100801.

\section{REFERENCES}

[1] W. Chen, Y. Liu, H. S. Courtney, M. Bettenga, C. M. Agrawal, J. D. Bumgardner and J. L. Ong, "In Vitro AntiBacterial and Biological Properties of Magnetron CoSputtered Silver-Containing Hydroxyapatite Coating," Biomaterials, Vol. 27, 2006, pp. 5512-5517. http://dx.doi.org/10.1016/j.biomaterials.2006.07.003

[2] M. Jelinek, M. Weiserová, T. Kocourek, K. Jurek and J. Strnad, "Doped Biocompatible Layers Prepared by Laser," Laser Physics, Vol. 20, No. 3, 2010, pp. 562-567. http://dx.doi.org/10.1134/S1054660X10050087 